\title{
WHEN IS THE SEMIGROUP RING PERFECT?
}

\author{
JAN OKNIŃSKI
}

ABSTRACT. A characterization of perfect semigroup rings $A[G]$ is given by means of the properties of the ring $A$ and the semigroup $G$.

It was proved in [10] that for a ring with unity $A$ and a group $G$ the group ring $A[G]$ is perfect if and only if $A$ is perfect and $G$ is finite. Some results on perfectness of semigroup rings were obtained by Domanov [3]. He reduced the problem of describing perfect semigroup rings $A[G]$ to checking that certain semigroup algebras derived from $A[G]$ satisfy polynomial identities. Further, a characterization of such $P I$-algebras over a field of characteristic zero was found in [2]. However, the obtained results are difficult to formulate and refer to some exterior constructions obscuring an insight into the properties of the semigroup.

The purpose of this paper is to completely characterize perfect semigroup rings by means of the properties of the semigroup and the coefficient ring. Our approach is quite different from that of [3] and omits $P I$-methods. It works in arbitrary characteristic and the final result is a natural strengthening of the conditions for $A[G]$ to be semilocal [7].

In what follows $A$ will be an associative ring, $G-$ a semigroup. $A$ is said to be right perfect if it is semilocal with its Jacobson radical $J(A)$ (right) $T$-nilpotent (cf. [4]). By $E(G)$ we shall mean the set of idempotents of $G$. If $e \in E(G)$, then we put $G_{e}=\{g \in e G e \mid g$ is invertible in $e G e\}$.

In the sequel the following well-known facts on $T$-nilpotence and perfectness will be useful.

Lemma 1 (cf. $[3,4])$. 1. If $G$ is a nil semigroup with d.c.c. on right principal ideals, then $G$ is T-nilpotent.

2. If $H$ is an ideal in $G$ and $G$ has d.c.c. on right principal ideals, then the semigroups $H, G / H$ also have d.c.c. on right principal ideals.

3. If $H$ is an ideal in $G$, then $A[G]$ is perfect if and only if so are the rings $A[H]$, $A[G / H]$.

By arguments similar to those from [7, Theorem 1], one can easily get

Proposition 1. Let $K$ be a field. Assume that $K[G]$ is perfect. Then the $K$-algebra $K[G] / J(K[G])$ is finite dimensional.

Received by the editors December 22, 1982.

1980 Mathematics Subject Classification. Primary 16A51, 20M25. 
We will start with the following

Lemma 2. Let $G$ be a periodic semigroup. Assume that $E(G)=E_{1} \cup \cdots \cup E_{s}$ with efe $\in G_{e}$ for any e, $f \in E_{i}$. Then there exists a chain of ideals $H_{1} \subset H_{2} \subset \cdots \subset H_{n}=$ $G$ such that $H_{1}, H_{i+1} / H_{i}, i>1$, are nil or completely 0 -simple.

Proof. We may assume that $G$ has a zero element $\theta$. Let $H_{1}$ be the largest nil ideal in $G$ and $G_{1}=G / H_{1}$. By hypothesis $G_{1} e G_{1}=G_{1} f G_{1}$ for any $e, f \in E_{i}, i=1, \ldots, s$. Thus $G_{1}$ has a minimal ideal of the form $G_{1} e G_{1}, e \in E(G) \backslash\{\theta\}$. Since $G_{1} e G_{1}$ has no nonzero nil ideals, then it is easily verified to be a 0 -simple semigroup. Moreover, any decreasing chain of idempotents in $G$ has length $\leqslant s$, and so $G_{1} e G_{1}$ is completely 0 -simple (cf. [1]). Hence, the required chain of ideals in $G$ may be built by continuing the above procedure.

Let $p$ be a prime number or 0 . In the latter case, by a $p$-group we shall mean the trivial group. The elements $e, f \in E(G)$ will be said to be $p$-equivalent if for any $g \in G$ the following statement holds:

ege $\in G_{e}$ if and only if efge, egfe $\in G_{e}$ and if it is the case, then ege, efge, egfe belong to the same coset of a normal $p$-subgroup in $G_{e}$.

THEOREM 1. Let $K$ be a field with char $K-p$. Then $K[G]$ is perfect if and only if

1. $G$ is periodic,

2. G has d.c.c. on right principal ideals,

3. $G$ has no infinite subgroups,

4. $E(G)=\cup_{i=1}^{s} E_{i}$ for some disjoint subsets $E_{i}$ of mutually p-equivalent idempotents.

Proof. Assume first that $K[G]$ is perfect. Then $G$ is periodic by [6]. Since the ring $K[G]$ has d.c.c. on right principal ideals [4], then $G$ satisfies the condition 2. Let $H$ be a subgroup in $G$. If $e=e^{2} \in H$, then $K[e G e]=e K[G] e$ is perfect [4]. It is easy to check that $K\left[G_{e}\right]$ is a direct summand of the left $K\left[G_{e}\right]$-module $K[e G e]$, hence it is perfect [8]. Thus, the group $G_{e}$ is finite [10], and so $H \subset G_{e}$ is finite.

Since $J(K[G])$ is $T$-nilpotent, then by Proposition $1 K[G]$ is a locally finite $K$-algebra. Thus, condition 4 follows from the fact that $K[G]$ is semilocal and from [7].

Assume now that $G$ satisfies conditions 1-4. Then, by Lemma 2, we have a chain of ideals $H_{1} \subset H_{2} \subset \cdots \subset H_{n}=G$ with $G_{1}=H_{1}, G_{i}=H_{i} / H_{i-1}, i=2, \ldots, n$, being nil or completely 0 -simple. Moreover, all $G_{i}$ inherit the assumptions on $G$. In the former case $G_{i}$ is $T$-nilpotent by Lemma 1 and hence $K\left[G_{i}\right]$ is perfect. If $G_{i}$ is completely 0-simple, then $G_{i} \simeq M^{0}\left(X^{0}, I, \Delta, P\right)$ is a Rees matrix semigroup over a group with zero $X^{0}$ with sandwich matrix $P[1]$. By $2 X$ is a finite group which easily implies that $G_{i}$ is locally finite. Now, it follows from [7] that $K\left[G_{i}\right]$ is semilocal. We will check that $J\left(K\left[G_{i}\right]\right)$ is nilpotent. If $e \subset E\left(G_{i}\right) \backslash\{\theta\}$, then $G_{i} e G_{i}=G_{i}$ and $J\left(K\left[G_{i}\right]\right)^{2} \subset K\left[G_{i}\right] J\left(K\left[G_{i}\right]\right)=K\left[G_{i}\right] e K\left[G_{i}\right] J\left(K\left[G_{i}\right]\right) \subset K\left[G_{i}\right] e J\left(K\left[G_{i}\right]\right)$. Now $e G_{i} e \simeq X^{0}[1]$, is finite. Thus $e J\left(K\left[G_{i}\right]\right) e=J\left(K\left[e G_{i} e\right]\right)$ is nilpotent from which follows the nilpotency of $J\left(K\left[G_{i}\right]\right)$.

Since $K\left[G_{i}\right]$ is perfect for any $i=1, \ldots, n$, it then follows from Lemma 1 that $K[G]$ is perfect. 
The necessity of condition 2 was proved in [3]. The fact that $K[G]$-perfect implies $G$-locally finite may be also deduced, in a different way, from [3].

Let us notice that, under the hypotheses of Theorem 1, there is a bound on cardinalities of subgroups in $G$. More precisely, for $e, f \in E_{i}$, let us consider the transformations $\varphi: e G e \rightarrow f G f, \psi: f G f \rightarrow e G e$ defined by $\varphi(x)=f x f . \psi(y)=e y e$. It may be verified that $\varphi\left(G_{e}\right) \subset G_{f}, \psi\left(G_{f}\right) \subset G_{e}$. Since some power of $\varphi \psi$ is the identity transformation, then the groups $G_{e}, G_{f}$ have the same number of elements. Moreover, if char $K=0$, then $\varphi$ is an isomorphism with $\varphi^{-1}=\psi$.

The following example shows that there is an essential difference between the conditions for $K[G]$ to be perfect in distinct characteristics (that is not the case for the class of group rings).

EXAMPLE. Let $I$ be an infinite set and $G=M(X, I, I, P)$ be a completely simple Rees matrix semigroup over a finite nontrivial $p$-group $X, p>0$. Then $K[G]$ is local for any field $K$ of characteristic $p$ [7]. Moreover, as in the proof of Theorem 1, $J(K[G])$ is nilpotent and hence $K[G]$ is right and left perfect. Let $e$ be the unity in $X$. Choose $e \neq g \in G$ and put $P=\left(p_{i j}\right), p_{i i}=e, p_{i j}=g$ for $i, j \in I, i \neq j$. Then $(e)_{i i} \in E(G)$ and $(e)_{i i}(e)_{k k}(e)_{j j}(e)_{i i}=\left(g^{3}\right)_{i i} \neq\left(g^{2}\right)_{i i}=(e)_{i i}(e)_{k k}(e)_{i i}$ for distinct elements $i, j, k \in I$. Thus, the idempotents $(e)_{i i}$. $(e)_{j, j}$ cannot be $q$-equivalent for $q \neq p$ and $L[G]$ is not semilocal for any field $L$ with char $L \neq p$.

It is known that if a ring $A$ is $T$-nilpotent, then for any sequence of finite subsets $A_{1}, A_{2}, \ldots$ in $A$ there exists $n>1$ such that $A_{1} A_{2} \cdots A_{n}=0$ [9]. Thus $A[G]$ is $T$-nilpotent for any semigroup $G$. Now, for arbitrary $A, A[G]$ is perfect if and only if so is $A / J(A)[G]$. Hence, the case of an arbitrary coefficient ring may be easily derived from the following

TheOREM 2. Let $A$ be an algebra over a field $K$. Assume that $A$ is not T-nilpotent. Then $A[G]$ is perfect if and only if so are the rings $A, K[G]$.

Proof. Since $A[G] \simeq A \otimes_{K} K[G]$, then the result follows from Proposition 1 and from [5, Theorem 1.4 and Theorem 2.5].

\section{REFERENCES}

1. A. H. Clifford and G. B. Preston, The algebraic theory of semigroups, Vol. I, Math. Surveys, No. 7. Amer. Math. Soc., Providence, R. I., 1961.

2. O. I. Domanov, On identities for semigroup rings of completely 0 -simple semigroups, Mat. Zametki 18 (1975), 203-212. (Russian)

3. _. Perfect semigroup rings, Sibirsk. Mat. Ž. 18 (1977), 294-303. (Russian)

4. C. Faith, Algebra. II, Ring theory, Springer-Verlag, New York, 1976.

5. J. Krempa and J. Okniński, Semilocal, semiperfect and perfect tensor products, Bull. Acad. Polon. Sci. 28 (1980), 249-256.

6. J. Okniński, Artinian semigroup rings, Comm. Algebra 10 (1982), 109-114.

7. __ Semilocal semigroup rings, Glasgow Math. J. (in print).

8. J. K. Park, Artinian skew group rings, Proc. Amer. Math. Soc. 75 (1979), 1-7.

9. E. M. Patterson, On the radical of certain rings of nonfinite matrices, Proc. Roy. Soc. Edinburgh 66 (1961/62), 42-46.

10. S. M. Woods, On perfect group rings, Proc. Amer. Math. Soc. 27 (1971), 49-52.

INSTITUTE OF MATHEMATICS, UNIVERSity OF WARSAW, 00-901 WaRSAW, Poland 